\title{
The DIAGNOSER: A HyperCard tool for building theoretically based tutorials
}

\author{
BJÖRN B. LEVIDOW, EARL HUNT, and COLENE MCKEE \\ University of Washington, Seattle, Washington
}

\begin{abstract}
The DIAGNOSER is a HyperCard tutorial construction system based on a framework of naive physics conceptions and developed for high school classroom use. It assesses students' conceptions in a domain, and prescribes a course of action on the basis of that assessment. In addition, it collects data for instructional enhancement and theoretical investigations. It is sufficiently general to allow tutorials to be developed in other knowledge domains, such as probability, economics, and psychology. An advanced version exploring naive conceptions of circuit electricity is also discussed.
\end{abstract}

Elementary physics is one of the most challenging courses offered in high school. In this course, students have to learn not only facts, but also new, systematic ways of reasoning about the physical world. At Mercer Island High School in the state of Washington, James Minstrell and his colleagues have developed a very successful course that stresses the conceptual basis of physics. As an adjunct to that course, students interact with the DIAGNOSER, a computer program intended to assist them in thinking about their own thinking.

Technically, the DIAGNOSER is a program for building theoretically based tutorials in the HyperCard environment. It allows instructors with little computer experience to design tutorials that (1) give individualized instruction to a student on the basis of a diagnosis of the student's naive conceptions in a content domain, and (2) collect data about students' naive conceptions. ${ }^{1}$ These data can be used to prescribe individual lesson plans and to conduct theoretical investigations of knowledge representation and problem solving.

\section{Theoretical Foundations}

The DIAGNOSER is based on James Minstrell's view of students' naive conceptions of physics (Minstrell \& Stimpson, 1990); Minstrell's ideas are similar to diSessa's knowledge-in-pieces model (diSessa, 1987, 1988). The basic view is that students bring to the classroom salient, incomplete, but not necessarily incorrect, conceptions about physical systems. ${ }^{2}$ These are called facets of knowledge.

The instructor first tries to identify the facets a student uses; the instruction of the student is then based on those

\footnotetext{
This work was supported by a research grant from the McDonnel Foundation to James Minstrell and Earl Hunt. We gratefully acknowledge the help and guidance of James Minstrell, Virginia Stimpson, Dorothy Simpson, Elizabeth Sanocki, John Dunlosky, Susan Infield, Dawn Aiken, and Emily van Zee. Correspondence concerning this article, or the described programs, should be addressed to Björn B. Levidow, Department of Psychology, University of Washington, Seattle, WA 98195.
}

facets. The goal is to make the student accept, not just recite, the conceptions of modern physics. The course puts more stress on attitudinal change than on didactic instruction. Preliminary data indicate that this instructional approach to physics is more effective than others (Minstrell \& Stimpson, 1990).

If this system is to work, the instructor must know what beliefs a particular student has. We call the elicitation of these beliefs diagnosis. Minstrell has developed a taxonomy of the different beliefs that he and his colleagues have observed. An example would be the fairly common misconception that stationary objects do not exert force. The DIAGNOSER poses special questions to students, as it "looks for" facets that have been defined by experienced teachers. It observes the students' answers and asks the students to explain their reasoning.

\section{DIAGNOSER Structure}

The student initially sees a list of instructional topics; the student chooses a topic by clicking on it with the mouse. The student is then presented with a multiplechoice question in that area. The answer choices are designed so that each alternative indicates the use of a different facet. An example is shown in Figure 1.

In the example question, choosing Answer $\mathrm{A}$ indicates that the student is using a facet that defines average velocity as a function of the final position and time, without regard for initial position. Answer B indicates that the student is using a facet that defines average speed as equivalent to average velocity. Upon choosing an answer, the student is asked to rate his or her confidence in that answer on a 6-point scale, from $0 \%$ to $100 \%$. Next, the student is asked to identify the reasoning processes he/she used to answer the initial question. For example, after rating his or her confidence in the answer to the question in Figure 1, the student is presented with Figure 2. The student has the ability to review the initial question by clicking on the "Review Question" button in Figure 2 .

At the end of the questioning, the student receives a message. The text is based on both the correctness of the 


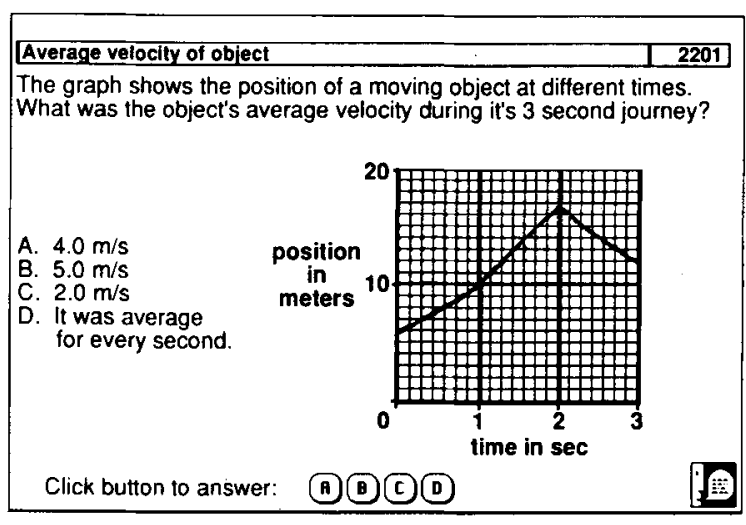

Figure 1. An example of the initial portion of a DIAGNOSER question.

answers to the initial question and the answer to the reasoning portion. For example, if a student has chosen an incorrect answer (i.e., $\mathrm{A}$ in the example) on the initial portion and a correct answer on the reasoning portion (i.e., B), the feedback would be the following:

Your reasoning seems okay, but you need to take a closer look at how you answered the question. Move ahead and get a prescription for help with the question. Work to make your answer consistent with your reasoning.

When the student has indicated that he or she is finished reading the feedback, a prescription is displayed. An example prescription is shown below.

Think back to the turtle problem from Motion 1. Did you get the same answer for average speed and average velocity? For average speed the turtle wants to get credit for the total distance her feet travelled. For average velocity all we care about for our calculation of change in position are the final and initial positions. Make up a problem situation that distinguishes between the average speed and average velocity, and show it to your teacher.

The students can use a primitive electronic mail system to leave notes for the instructor. Notes can be requested by the instructor, as is shown by Answer E in Figure 2. The students may also write about difficulties

\footnotetext{
The average velocity is

A. the final position divided by the final clock reading

$B$. the change in position divided by the change in clock reading.

C. constant only curing each one second interval.

D. the total distance traveled divided by the total elapsed time.

E. Other (Please explain on a notecard).

Click button to answer: $A(B)$ (B) Review question
}

Figure 2. An example of the reasoning portion of a DIAGNOSER question. that they are having with certain concepts or with the operation of the tutorial. After answering all three portions of a question, the student has four options: (1) review the same question, (2) see another question on the same topic, (3) return to the topic list to select a new topic, or (4) quit the tutorial.

\section{Facet Construction}

Questions, possible answers, and facets are defined by experienced instructors. The DIAGNOSER itself has no knowledge of physics. The program associates two parameters with each facet: a correctness value, and a prescription. The correctness value indicates whether or not the facet agrees with an expert's reasoning. The prescription is a message displayed to the student in an attempt to shape the student's understanding of a topic to be more like that of an expert.

The correctness value is used by the tutorial to determine which of five feedback messages should be presented to the student. The message reflects the correctness of the student's answers to both the initial and the reasoning portions of the question. The agreement, or lack thereof, between the facet assignments is also reflected in the message.

The prescription is displayed once the tutorial has diagnosed the student as using a particular facet. The diagnosis is made by comparing the facet assigned to the answer for the initial portion of the question (initial facet) with the facet assigned to the answer for the reasoning portion of the question (reasoning facet). Usually, the prescription stored in the initial facet will be displayed. However, if there is no agreement between the facets and the initial facet is correct, the prescription in the reasoning facet will be displayed. In general, the prescription instructs the student to consider specific issues, review problems presented in class, read pages in the text, and/or see the instructor for clarification.

\section{Question Construction}

Once the facets have been developed, questions can be constructed to differentiate between different facets. Both the initial and the reasoning portions of a question are multiple-choice, with up to six answers per portion. In the initial portion, questions are asked directly about the phenomenon of interest. The question is placed into the DIAGNOSER with the HyperCard text and drawing tools. After the appearance of the question is to the instructor's liking, the DIAGNOSER prompts the instructor to enter the facet associated with each answer.

In the reasoning portion, the instructor designs questions about the reasoning processes that the students use to arrive at their initial answer. There are three motivations for having this portion in the DIAGNOSER. First, research indicates that by making their reasoning process explicit, students are forced to reevaluate the knowledge they have used to reach a particular conclusion (Chi, Bas- 
sok, Lewis, Reimann, \& Glaser, 1989). Second, over the last year and a half, students have indicated that they enjoy this portion of the question and find it useful. Third, a student's answers to the initial and reasoning portions of a question are compared to achieve a more accurate facet diagnosis. To ensure that this comparison can be made, answers to the initial and reasoning portions of the question should both be assigned to the same facets. For example, Answer D in Figure 1 and Answer C in Figure 2 are both assigned to the facet that defines velocity as equivalent to average velocity.

Answers may be assigned to facets that have not yet been placed in the DIAGNOSER. This is done to keep the question-designing process flexible, but it can cause problems. If a student selects an answer without a facet assignment, an error message is displayed, and, of course, no prescription is given. To ensure that students always receive a prescription, a utility has been provided to alert instructors when answers have not been assigned to facets. Ideally, the designer of a DIAGNOSER tutorial should begin by entering a group of facets in a particular knowledge domain, but the described structure makes this process optional.

\section{Design Considerations}

There were many considerations taken into account in the design of the DIAGNOSER in addition to the Minstrell framework. We wanted to maximize exportability, so that the DIAGNOSER could be used as a tool to construct classroom software. We achieved this by making the DIAGNOSER a framework with tools to allow tutorial customization. This flexibility allows DIAGNOSER tutorials to be easily integrated into most classroom situations.

An important objective in the DIAGNOSER's design was to gather data to be used by teachers and researchers while tutoring students in a content domain. The DIAGNOSER records the date, the time, the question asked, confidence ratings, and the facet number corresponding to the student's answers each time the student completes all three portions of a question.

The wide range of computer knowledge possessed by the instructors and students who design and use the DIAGNOSER was also one of our concerns. Because questions frequently contain both graphical and textual information, intuitive tools for editing both types of information were necessary. Therefore, the HyperCard environment was chosen to develop the DIAGNOSER, because these tools are an integral part of the environment. However, the disadvantage of this environment is that any program developed in HyperCard can be easily damaged by students or instructors.

To reduce this risk, special external commands (XCMDs) were combined with HyperScript functions to limit access to actions that could damage the program. Specifically, unavailable actions are either denoted by gray menu choices or reported by error message. In addition, great care has been taken to make the question-designing environment easy to use. The standard Macintosh interface has been preserved whenever possible. Informative error messages are provided, so that instructors need only concern themselves with the tutorial domain and not the DIAGNOSER environment itself.

The DIAGNOSER was designed to function on the lowcost, compact line of Macintosh computers (i.e., the Mac Classic) to minimize both the cost and the physical size of the hardware. This was a concern because both money and space are limited resources in high school classrooms. This contrasts with other cognitive diagnostic systems, such as that of Shute, Glaser, and Raghvan (1989), which require large, expensive hardware systems.

\section{The SuperDiagnoser: \\ An Advanced DIAGNOSER}

A specialized version of the DIAGNOSER program has also been developed specifically to construct tutorials in the domain of circuit electricity. This program, the SuperDiagnoser (SD), allows students more control over the issues they want to investigate. The student interacts with the SD tutorial to create his or her own electricity problem. The student can build a circuit of interest out of available elements (e.g., bulbs, batteries, resistors), displayed on a pop-up menu, in 13 fixed positions.

The SD will allow the student to make only circuits that have been assigned to questions by the instructor. It accomplishes this by limiting the elements available to the student, on the basis of the elements that the student has previously chosen. Unavailable elements are represented by gray items on the pop-up menu.

The instructor can assign up to six spatial variations of the same circuit to a particular question. Once the student has constructed a circuit, the question associated with that circuit will be presented. An example is shown in Figure 3.

The format of the question in the SD is different from that of the standard DIAGNOSER. The SD allows, but does not require, hypothetical or 'what if' situations to be dis-

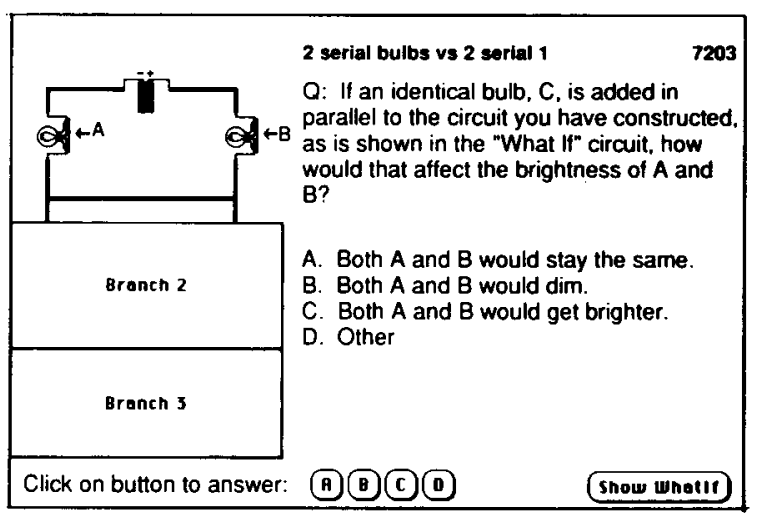

Figure 3. An example of the initial portion of a SuperDiagnoser question. 


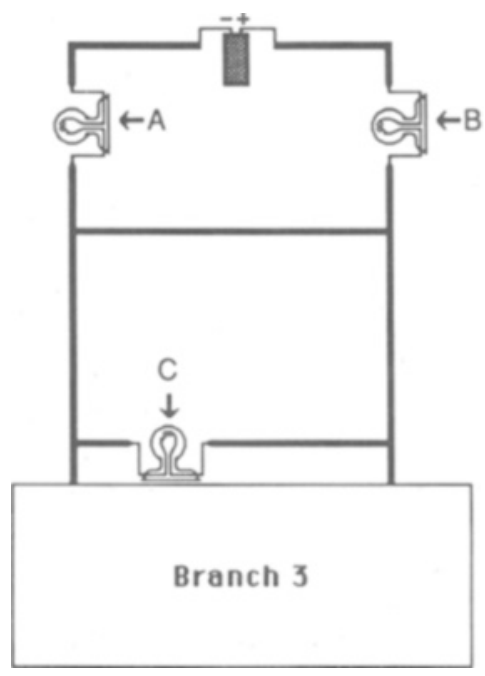

Figure 4. The "what if" display associated with the SuperDiagnoser question shown in Figure 3.

played along with the circuit constructed by the student. The student can alternate the circuit display between the circuit described by the "what if" question, shown in Figure 4, and the circuit that the student has constructed. After the student has answered the initial question, the SD format is identical to that of the DIAGNOSER tutorials.

\section{Applications}

The DIAGNOSER is a general implementation of Minstrell's instructional approach, and it is not tied directly to physics. Owing to their ease of use and low-cost hardware requirements, DIAGNOSER tutorials can be made an integral part of many classroom settings. As a result, the DIAGNOSER could be used to develop tutorials within a range of knowledge domains, such as economics, statistics, and some areas of psychology (e.g., the visual system, neurochemistry).

The data collected by the DIAGNOSER tutorials can be used to monitor the day-to-day progress of students. The data can also be used to determine the naive conceptions students still possess after instruction. This allows instructors to tailor their lessons to the actual state of student knowledge. In addition, the data may also be used to gain a better understanding of the changes that occur in a student's knowledge state as the student receives instruction.

The confidence portion of the question was placed in the DIAGNOSER tutorials to allow analysis techniques used in the feeling-of-confidence literature to be applied to tutorial data (for a review, see Lichtenstein, Fischhoff, \& Phillips, 1982). Questions such as, "Is there a relationship between the degree of confidence a student has in a facet and the susceptibility of that facet to instruction?" are currently being investigated with the use of DIAGNOSER tutorial data.

Future versions of the DIAGNOSER will allow greater flexibility in the operation of the tutorials. Currently, every question presented to the student is randomly chosen from the instructional area that the student has selected. In the future, instructors will be able to select two additional manners of presentation: linear and contingent. Linear presentation will display questions to the students in an order predetermined by the instructor. Contingent presentation will display questions to students on the basis of their answers to previous questions. We hope that through the addition of flexibility, the DIAGNOSER will become more attractive for use in both instructional and research environments.

\section{REFERENCES}

Chi, M. T. H., Bassok, M., Lewis, M. W., Reimann, $P_{\text {, }}$ \& Glaser, R. (1989). Self-explanations: How students use examples in learning to solve problems. Cognitive Science, 13, 145-182.

DISESSA, A. A. (1987). Toward an epistemology of physics (Tech. Rep. No. 48). University of California, Berkeley, Institute for Cognitive Science.

DISessa, A. A. (1988). Knowledge in pieces. In G. Forman \& P. Puffall (Eds.), Constructivism in the computer age (pp. 49-70). Hillsdale, NJ: Erlbaum.

Lichtenstein, S., Fischhoff, B., \& Phillips, L. D. (1982). Calibration of probabilities: The state of the ant to 1980. In D. Kahneman, P. Slovic, \& A. Tversky (Eds.), Judgement under uncenainty: Heuristics and biases (pp. 306-334). New York: Cambridge University Press. MINSTRELL, J., \& STIMPSON, V. (1990, April). A teaching system for diagnosing students' conceptions and prescribing relevant instruction. Paper presented at the meeting of the American Education Research Association, Boston, MA.

Shute, V., Glaser, R., \& Raghyan, K. (1989). Inference and discovery in an exploratory laboratory. In P. L. Ackerman, R. J. Sternberg, \& R. Glaser (Eds.), Learning and individual differences (pp. 279326). New York: W. H. Freeman.

\section{NOTES}

1. A naive conception is an idea about the workings of a system, developed in the absence of formal training.

2. Conceptions of correctness change as different theories in a domain gain and lose acceptance. For example, it was once thought that combustion was caused by the release of a particular substance, phlogiston, into the atmosphere. 